\title{
Estudo comparativo sobre a aquisição de palavras de classe aberta e classe fechada em crianças com desenvolvimento típico de linguagem
}

\section{Comparative study on the acquisition of open class words and closed class in children with typical language development}

\section{Estudio comparativo sobre la adquisición de palabras de clase abierta y clase cerrada en niños con desarrollo típico de lenguaje}

\author{
Letícia Arruda Nóro* \\ Diogo Ribas dos Santos* \\ Renata de Quadros Machado* \\ Karine Rodrigues Freitas* \\ Gabriela Brum dos Santos* \\ Fernanda Marafiga Wiethan* \\ Helena Bolli Mota*
}

\section{Resumo}

Introdução: $\mathrm{O}$ aumento do vocabulário é um aspecto de grande importância para o desenvolvimento cognitivo da criança. Abrange vários aspectos das palavras como suas pronúncias, as definições de seus significados, regras sintáticas de seus usos e o modo como as palavras são escritas. Objetivo: verificar a aquisição de palavras de classe aberta e fechada em crianças com idades entre 2 anos e 4 anos, 11 meses

*Universidade Federal de Santa Maria, UFSM - Santa Maria, RS, Brasil

Contribuição dos autores:

LAN: foi responsável pelo delineamento do estudo, coleta e elaboração do banco de dados; DRS, RQM, KRF e GBS: análise dos resultados, levantamento bibliográfico da literatura e elaboração do manuscrito; FMW: elaboração e revisão crítica; HBM: elaboração e revisão crítica

E-mail para correspondência: Letícia Arruda Nóro - titamecmat@gmail.com Recebido: 21/01/2019

Aprovado: 24/09/2019 
e 29 dias, matriculadas em escolas de educação infantil da rede pública municipal de de Santa Maria. Métodos: a amostra foi composta por 72 crianças com idades entre dois e quatro anos, 11 meses e 29 dias, sendo 36 meninos e 36 meninas, distribuídos uniformemente entre faixas etárias, com desenvolvimento típico de linguagem. Foram realizadas filmagens da fala espontânea de cada sujeito, e após, realizou-se a análise do vocabulário. Para análise estatística foram utilizados o Programa Statistical Analysis System, versão 9.2 e os testes de Mann-Whitney e Kruskal-Wallis. Em ambos os testes o nível de significância adotado foi de $5 \%(\mathrm{p}<0,05)$. Resultados: não houve influência do sexo no desempenho de aquisição de classes de palavras. No entanto, quanto à faixa etária, pode ser observada diferença estatisticamente significante na aquisição de palavras de classe fechada. Conclusão: de acordo com os resultados, foi possível concluir que a aquisição de palavras de classe aberta não é influenciada pelas variáveis sexo e idade. Houve relação positiva entre aquisição de palavras de classe fechada e a faixa etária, sendo maior nas crianças com maior idade.

Palavras-chave: Vocabulário; Desenvolvimento da Linguagem; Desenvolvimento Infantil; Fala; Criança.

\section{Abstract}

Introduction: The increasing of vocabulary is an aspect of great importance for the child's cognitive development. Covering various aspects of words as their pronunciations, definitions of their meanings, syntactic rules of their uses and the way words are written. Purpose: to investigate the acquisition of open and closed class words in children aged 2 years to 4 years, 11 months and 29 days, enrolled in preschools within the municipal public network of Santa Maria. Methods: the sample was composed of 72 children aged from two to four years, 11 months and 29 days, being 36 boys and 36 girls, with typical language development, distributed evenly among age groups. Filming of spontaneous speech of each subject was held, and after, was held the analysis of the vocabulary and mean length of utterance. For statistical analysis was used the Statistical Analysis System, 9.2, and Mann-Whitney and Kruskal-Wallis tests, with significance level $p<0.05$. Results: it was not observed influence of gender in performance acquisition of speech. However, as to the age group, it can be observed significant difference in the acquisition of closed-class words. Conclusion: According to the results, it was concluded that the acquisition of open class words is not influenced by gender and age variables. There was a positive relationship between acquisition of closed-class words and the age range, being higher in children with higher age.

Keywords: Vocabulary; Language Development; Child Development; Speech, Child

\section{Resumén}

Introducción: El aumento del vocabulario es un aspecto de gran importancia para el desarrollo cognitivo del niño. Abordando varios aspectos de las palabras como sus pronunciaciones, las definiciones de sus significados, reglas sintácticas de sus usos y el modo en que se escriben las palabras. Objetivo: verificar la adquisición de palabras de clase abierta y cerrada en niños con edades entre 2 años a 4 años, 11 meses y 29 días, matriculadas en escuelas de educación infantil de la red pública municipal de Santa Maria. Métodos: la muestra fue compuesta por 72 niños con edades entre dos años a cuatro años, 11 meses y 29 días, siendo 36 niños y 36 niñas, distribuidos uniformemente entre franjas etarias, con desarrollo típico de lenguaje. Se realizaron filmaciones del habla espontánea de cada sujeto, y después, se realizó el análisis del vocabulario. Para el análisis estadístico se utilizó el Programa Statistical Analysis System, versión 9.2 y las pruebas de Mann-Whitney y Kruskal-Wallis. En ambas pruebas el nivel de significancia adoptado fue del 5\% $(\mathrm{p}<0,05)$. Resultados: no hubo influencia del sexo en el desempeño de adquisición de clases de palabras. Sin embargo, en cuanto al grupo de edad, puede observarse una diferencia estadísticamente significativa en la adquisición de palabras de clase cerrada. Conclusión: de acuerdo con los resultados, fue posible concluir que la adquisición de palabras de clase abierta no está influenciada por las variables de género y edad. Hubo relación positiva entre adquisición de palabras de clase cerrada y el grupo de edad, siendo mayor, en los niños con mayor edad.

Palabras clave: vocabulario; Desarrollo del Lenguaje; Desarrollo Infantil; habla; niño 


\section{Introdução}

Um dos índices para monitorar o desenvolvimento de linguagem é a produção do vocabulário. O conhecimento e o aumento do vocabulário são aspectos de grande importância para o desenvolvimento cognitivo da criança. Eles contribuem para o bom resultado da leitura e compreensão de palavras, e têm implicações no processo de aprendizagens posteriores no decorrer do ensino escolar, por exemplo ${ }^{1-4}$.

Este conhecimento abrange vários aspectos das palavras, como suas pronúncias, as definições de seus significados, regras sintáticas de seus usos e o modo como as palavras são escritas. Todos esses elementos estão conectados a uma rede de representações mentais, e a qualidade destas se relacionará com o bom uso do vocabulário ${ }^{5-7}$.

Pesquisa $^{8}$ afirma que existe influência do tipo de palavras na produção oral das crianças. Didaticamente, elas podem ser divididas em classe aberta ou fechada. As primeiras são consideradas palavras de conteúdo e apresentam significado lexical e, em princípio, sempre possibilitam novas criações. Pertencem a essa classe substantivos, adjetivos, verbos, advérbios e numerais, que exercem papel fundamental na transmissão da informação semântica.

A segunda classe refere-se às palavras funcionais (ou de classe fechada), que apresentam um significado gramatical. Elas são índices de propriedades gramaticais que fazem diferença entre as línguas. Essas palavras são os artigos, preposições, conjunções, pronomes e interjeições, as quais exercem principalmente função sintática, servindo como elementos de ligação frasal com baixa carga semântica própria.

Como exemplo de estudos sobre aquisição em crianças com desenvolvimento típico, uma pesquisa $^{9}$ analisou a mudança em types e tokens e na taxa de type/token em crianças, de ambos os sexos, entre 18 e 36 meses, falantes do português brasileiro, quanto à classe gramatical e à medida total e segmentar. Os resultados indicaram que os substantivos surgem primeiro do que verbos, advérbios e adjetivos e demais classes gramaticais que se completam até os 32 meses.

Outro trabalho ${ }^{10}$ verificou de que modo se dá a aquisição lexical inicial de crianças com desenvolvimento típico, em termos de tipos e ocorrências dos itens lexicais, e, ainda, se a hipótese do viés nominal realmente ocorre. Os resultados da análise indicaram que o número de substantivos foi superior ao dos verbos durante o período de aquisição lexical estudado, porém a produção de substantivos não foi exclusiva mesmo neste período bem inicial de aquisição linguística.

Além dessa pesquisa, outra também mostrou uma superioridade na aquisição dos substantivos, em que ocupou uma média de $55 \%$ do léxico das crianças com vocabulário entre 100 e 200 palavras, enquanto as palavras de conteúdo foram responsáveis por menos de $15 \%$.

Com base no exposto, o objetivo deste estudo foi verificar a aquisição de palavras de classe aberta e fechada em crianças com idades entre 2 anos e 4 anos, 11 meses e 29 dias, matriculadas em escolas de educação infantil da rede pública municipal de Santa Maria.

\section{Métodos}

Este estudo caracteriza-se como sendo de caráter quantitativo, descritivo e com coleta de dados transversal e faz parte de um projeto aprovado no Comitê de Ética em Pesquisa de uma instituição de ensino superior em cidade do interior do Rio Grande do Sul, sob o número do CAEE: 0219.0.243.00011. Como condição obrigatória para participação do estudo, os responsáveis pelos sujeitos assinaram o Termo de Consentimento Livre e Esclarecido.

A amostra foi composta por 72 crianças com idades entre dois anos e quatro anos, 11 meses e 29 dias, sendo 36 meninos e 36 meninas, distribuídos uniformemente entre faixas etárias. Todas as crianças eram membros de famílias monolíngues falantes do Português Brasileiro, com desenvolvimento típico de linguagem. As crianças que apresentassem perda auditiva, comprometimento neurológico, emocional e/ou cognitivo, detectável por meio de observação; presença de alterações motoras ou orgânicas orais, ou crianças que tivessem realizado ou estivessem realizando fonoterapia, foram excluídas da pesquisa.

A coleta dos dados foi realizada em escolas municipais de Educação Infantil de uma cidade do interior do Rio Grande do Sul. A avaliação fonoaudiológica incluiu questionário destinado aos responsáveis, avaliação orofacial e das praxias orais, avaliação da linguagem oral e triagem auditiva.

Para avaliação dos aspectos orofaciais utilizou-se o "Protocolo de avaliação miofuncional 
orofacial com escores (AMIOFE) ${ }^{11}$ " adaptado. Para as crianças com idade superior a três anos e seis meses, utilizou-se adicionalmente o Protocolo de avaliação da dispraxia ${ }^{12}$ para verificação das praxias orais.

A avaliação da linguagem foi realizada por meio do Protocolo de Observação Comportamental (PROC) ${ }^{13}$. Desse modo, foi possível observar o desenvolvimento de linguagem e cognitivo, quanto aos aspectos semânticos, sintáticos e pragmáticos. Assim, pode-se avaliar os comportamentos motor e social. O PROC foi construído para crianças de um a quatro anos de idade, é de fácil aplicação e contém valores de referência definidos. Com as crianças maiores, empregou-se narrativas orais espontâneas. Além disso, os aspectos fonéticos e fonológicos da fala foram observados em conversação espontânea e repetição de vocábulos foneticamente balanceados.

As crianças que contemplaram os critérios de inclusão procederam-se a avaliação do vocabulário, realizada por meio de fala espontânea e nomeação de objetos e brinquedos em miniatura. Foram realizadas gravações em vídeo durante 20 minutos para posterior transcrição fonética ampla da fala da criança e transcrição alfabética da fala da examinadora. Para a transcrição da fala das crianças até 3 anos 3 meses e 29 dias, utilizou-se o método do consenso ${ }^{14,15}$, ou seja, duas julgadoras trabalharam independentemente na transcrição; após, as transcrições foram comparadas e as discrepâncias ouvidas, novamente, por uma terceira julgadora até chegarem à concordância em todos os enunciados/ palavras/sons produzidos pela criança. Caso não houvesse a concordância entre pelo menos duas julgadoras, o trecho era excluído.

Com as crianças das demais faixas etárias, que apresentam as produções mais estáveis, utilizou-se o método de confiabilidade ${ }^{16,17}$ entre as transcrições.
Deste modo, para análise do vocabulário, foram utilizados os seguintes critérios: "tipos e ocorrências" ou "types e tokens" e classe aberta e classe fechada de palavras.

Tipos e ocorrências ou types e tokens: a fala das crianças foi separada por palavras, sendo contabilizados os tipos e as ocorrências (types e tokens) de cada sujeito. Para a classificação dos tipos, foram considerados o número dos diferentes itens lexicais produzidos pela criança. A contagem das ocorrências seguiu os mesmos critérios, a partir da identificação do número de repetições de cada tipo de palavra no corpus. Assim, podem ser verificadas as frequências de produção de cada classe de palavras: classe aberta (palavras de conteúdo): substantivos, adjetivos, verbos, advérbios e numerais; classe fechada (palavras funcionais): artigos, preposições, conjunções, pronomes, interjeições.

Utilizou-se o Programa Statistical Analysis System, versão 9.2 para realizar o teste de Mann-Whitney para comparação da classe aberta e classe fechada entre os sexos e, o teste de Kruskal-Wallis para comparação da classe aberta e classe fechada entre as faixas etárias. Em ambos os testes, o nível de significância adotado foi de $5 \%(\mathrm{p}<0,05)$.

\section{Resultados}

O desempenho da classe de palavras de classe aberta e classe fechada, bem como a comparação entre as faixas etárias podem ser visualizados na Tabela 1. Verificou-se diferença estatisticamente significante entre as faixas etárias para palavras de classe fechada.

A Tabela 2 apresenta o desempenho de palavras de classe aberta e fechada com relação ao sexo. Os resultados indicam que não há diferença estatisticamente significante para as variáveis.

Tabela 1. Análise comparativa das classes de palavras entre as faixas etárias

\begin{tabular}{lcccc}
\hline & 2 anos & 3 anos & 4 anos & Valor de P \\
\hline Classe Aberta & 196,29 & 223,58 & 227,04 & 0,147 \\
Classe Fechada & 89,92 & 129,29 & 160,17 & $<0,001$ \\
\hline
\end{tabular}

*Teste estatístico utilizado: Kruskal-Wallis. Foram considerados estatisticamente significantes os valores de $p<0,05$. 
Tabela 2. Análise comparativa das classes de palavras entre os sexos

\begin{tabular}{ccc}
\hline & Média da CA & Média da CF \\
\hline Feminino & 227,42 & 135,39 \\
Masculino & 203,86 & 117,53 \\
Valor de P* & 0,262 & 0,161 \\
\hline
\end{tabular}

*Teste estatístico utilizado: Mann-Whitney. Foram considerados estatisticamente significantes os valores de $\mathrm{p}<0,05$. Legenda: $\mathrm{CA}=$ classe aberta; $\mathrm{CF}=$ classe fechada.

\section{Discussão}

Pelos resultados encontrados na Tabela 1 , pode-se verificar que o aumento da idade parece implicar em um maior uso das palavras de classe fechada. Isso devido a uma necessidade maior de refinar as representações fonológicas e lexicais, indicando alta relação.

Os resultados deste estudo corroboram a uma pesquisa recente, que teve por objetivo observar a aquisição das primeiras palavras pelas crianças, contrapondo a aquisição de palavras de classe aberta e fechada e observando a emergência das primeiras combinações de palavras. Nesta pesquisa os autores puderam observar que o número de classes fechadas aumenta consideravelmente ao longo do tempo ${ }^{18}$.

Isso confirma estudos ${ }^{19,20}$ que mostram uma tendência inicial para a produção de classes abertas, as que têm um referente no mundo, se ampliando em seguida para a produção de classes fechadas, as que têm funções especificamente gramaticais, permitindo à criança construir a sintaxe da sua língua. Relacionando ao presente estudo, compreende-se que as crianças adquirem as palavras de classe aberta precocemente, por isso há um número considerável dessas palavras logo no início da aquisição (por volta dos dois anos), havendo aumento mais lento, porém constante após esse período. Já as palavras de classe fechada, estão relacionadas à sintaxe, mais complexa, e, portanto, ainda não estabilizada mesmo na idade de 4 anos, precisando ainda de muito refinamento em termos de aquisição das palavras mais complexas como as conjunções, por exemplo.

Conforme os resultados estatísticos, pode-se inferir que a aquisição inicial privilegia as classes abertas em detrimento das fechadas. Um estudo internacional ${ }^{21}$ mostrou que no início do processo de aquisição da linguagem as crianças têm maior compreensão em adquirir palavras de classe aberta, isso devido ao seu conteúdo mais concreto e seu maior uso na língua.

Conforme as crianças são expostas a novos itens lexicais, para que haja o aumento do vocabulário, elas começam a adquirir palavras de classe fechada, devido à exigência sintática de elementos de ligação frasal ${ }^{22}$.

Ainda, muitas hipóteses tentam explicar como ocorre a aquisição das categorias funcionais. Uma delas, a Hipótese Maturacional2 ${ }^{23}$, indica que as crianças não têm as categorias funcionais disponíveis na sua gramática mental. Já a outra hipótese, Hipótese Continuísta, defende a ideia de que as crianças têm essas categorias na sua gramática mental desde o nascimento ${ }^{24}$. O principal objetivo do presente estudo não abrange esta questão especificamente, mas é importante salientar para estudos futuros.

É importante analisar a relação que a memória e a linguagem estabelecem durante a infância. Antes do primeiro ano de vida, mecanismos já favorecem o aprendizado de palavras, aos 2 anos, há evidências de que informações linguísticas disponíveis à criança direcionam o armazenamento de informações da memória. Aos 3 anos, danos na memória de curto prazo verbal são associados à restrição do vocabulário e à produção de frases mais simples e curtas ${ }^{25-28}$.

Já na Tabela 2, não foram observadas distinções estatisticamente significantes entre meninos e meninas, demonstrando um equilíbrio evolutivo entre os grupos.

Em um estudo francês ${ }^{29}$, foi examinada a influência do sexo e fatores socioculturais da produção de linguagem de 316 amostras de fala de crianças, onde foram realizadas medidas de desenvolvimento gramatical e lexical, incluindo Extensão Média do Enunciado e relação type/token.

O resultado estatístico do estudo supracitado revelou intensa influência de fatores socioculturais, evidenciando que crianças de famílias de alto nível 
sociocultural têm produção lexical mais complexa e uma maior taxa de desenvolvimento.

\section{Conclusão}

A partir dos resultados é possível concluir que, para a população estudada, a aquisição de palavras de classe aberta não é influenciada pelas variáveis sexo e idade. Já a aquisição de palavras de classe fechada sofre influências da idade, ou seja, quanto maior a idade da criança, mais palavras gramaticais ela produz.

Estes resultados estão relacionados principalmente à sintaxe, porque é adquirida mais tardiamente pelas crianças por implicar na aquisição de palavras complexas, que só apresentam significado no contexto frasal.

\section{Agradecimentos}

Agradecemos ao CNPq e à CAPES pelo apoio concedido para a realização desta pesquisa.

\section{Referências}

1. Befi-Lopes DM, Galea DES. Análise do desempenho lexical em crianças com alteração no desenvolvimento da linguagem. Pró-Fono. 2000; 12(9): 31-7.

2. Befi-lopes DM, Puglisi ML, Rodrigues A, Giusti E, Gândara JP, Araújo K. Perfil comunicativo de crianças com Alterações Específicas no Desenvolvimento da Linguagem: caracterização longitudinal das habilidades pragmáticas. J Soc Bras Fonoaudiol. 2007; 12(4): 265-73.

3. Capovilla AGS, Capovilla FC, Soares JVC. Consciência sintática no ensino fundamental: correlações com consciência fonológica, vocabulário, leitura e escrita. Psico-USF. 2004; 9(1): 39-47.

4. Damazio M. Testes de vocabulário receptivo e expressivo: estudo de normatização e validação em crianças de 18 meses a 6 anos de idade [dissertação]. São Paulo (SP): Universidade de São Paulo;2011.

5. Kirby JR, Bowers PN. What works? research into practice. Student Achievement Division. 2012 ; 41(51): 1-4.

6. Bowers PN, Kirby JR, Deacon SH. The effects of morphological instruction on literacy skills: A systematic review of the literature. Rev Educ Res. 2010; 80(2): 144-79.

7. Kirby JR, Deacon SH, Bowers PN, Izenberg L, WadeWoolley L, Parrila R. Children's morphological awareness and reading ability. Read Writ. 2012; 25: 389-410.

8. Rosa MC. Classes de palavras, tipos de significado e questões relacionadas. In: Rosa MC. Introdução a morfologia. São Paulo:Contexto; 2003. p. 91-114.
9. Scherer S, Souza APR. Types e tokens na aquisição típica de linguagem por sujeitos de 18 a 32 meses falantes do português brasileiro. Rev CEFAC. 2011; 13(5): 838-46.

10. Nóro LA, Silva DD, Wiethan FM, Mota HB. Aquisição lexical inicial e verificação da hipótese do viés nominal. Rey CEFAC. 2015; 17(sup11):52-9.

11. Felício CMD, Folha GA, Gaido AS, Dantas MDMM, Azevedo-Marques P D. Protocolo de avaliação miofuncional orofacial com escores informatizado: usabilidade e validade. Revista Codas. 2014; 26(4): 322-7.

12. Hage SRV. Distúrbio específico do desenvolvimento da linguagem: subtipos e correlações neuroanatômicas. [tese] Campinas (SP): Faculdade de Ciências Médicas - UNICAMP; 2000 .

13. Zorzi JL, Hage SRV. PROC - Protocolo de observação comportamental: avaliação de linguagem e aspectos cognitivos infantis. São José dos Campos: Pulso Editorial; 2004.

14. Lidden G, Kankkonen A. Visual reinforcement audiometry. Acta OtoLaryngologica. 1969; 67(2-6):281- 92.

15. Morris SR. Test-Retest Reliability of Independent Measures of Phonology in the Assessment of Toddlers' Speech. Lang. speech hear. serv. sch. 2009; 40(1): 46-52.

16. McLeod S, Harrison LJ, McCormack J. The intelligibility in context scale: validity and reliability of a subjective rating measure. J Speech Lang Hear Res. 2012; 55(2): 648-56.

17. Sosa AV, Stoel-Gammon C. Lexical and Phonological Effects in Early Word Production. J Speech Lang Hear Res. 2012; 55(2): 596-608.

18. Passos AP, Costa IO, Salgado OFA, Haddad VCN. Adquirindo as primeiras palavras: categorias abertas e fechadas e as primeiras combinações. In: Bernardo S, Augusto MRA, Vasconcellos Z. Linguagem: teoria, análise e aplicações. $1^{\circ}$ ed. Rio de Janeiro: Programa de Pós Graduação em Letras; 2011. P.228-38.

19. Barret MD. Desenvolvimento lexical inicial. In: Fletcher P, Macwhinney B. Compêndio da linguagem da criança. $1^{\circ}$ edição. Porto Alegre: Artes Médicas; 1997. p. 299-322.

20. Dar M, Anwaar H, Vihman M, Keren-Portnoy T. Developing an Urdu CDI for early language acquisition. York Papers in Linguistics. 2015; 14(2): 1-14.

21. D'odorico L, Fasolo M. Nouns and verbs in the vocabulary acquisition of Italian children. J Child Lang. 2007; 34(4): 891-907.

22. Rizzi L. On the grammatical basis of language development: a case study to appear. In: G Cinque. Handbook of comparative sintax. New York: Oxford University Press; 2012.

23. Meisel J., Getting FAT: Finiteness, Agreement and Tense in Early Grammars. In: Meisel J. Bilingual First Language Acquisition: French and German Grammatical Development. Amsterdam: John Benjamins Publ. Co; 1994. P. 89-129.

24. Bishop DV. How does the brain learn language? Insights from the study of children with and without language impairment. Dev Med Child Neurol. 2000; 42(2): 133-42.

25. Benavides Varela S, Mehler J. Verbal positional Memory in 7 months olds. Child Dev. 2015,86(1): 209-23.

26. Haden CA, Ornstein PA, O'Brien BS, Elischberger HB, Tyler CS, Burchinal MJ. The development of children's early memory skills. J Exp Child Psychol. 2011; 108(1): 44-60. 
27. Clark E. Adult offer, word-class, and child uptake in early lexical acquisition. First Language. 2010; 30(3-4): 250-69.

28. Perry LK, Perlman M, Lupyan G. Iconicity in English and Spanish and Its Relation to Lexical Category and Age of Acquisition. PLoS ONE. 2015; 10(9): 1-17.
29. Le Normand MT, Parisse C, Cohen H. Lexical diversity and productivity in French preschoolers: developmental, gender and sociocultural factors. Clin Linguist Phon. 2008; 22(1): 47-58. 